at low degrees, merely broadened lines at higher degrees. There is no formal distinction between Rayleigh waves and Love waves on a spherical surface and free oscillations. For instance, the very long period "spheroidal" oscillations can be regarded alternatively as very long wavelength (a fraction of the Earth's circumference) Rayleigh waves.

Free oscillations of the Earth are obviously excited by the slightest tap but instrumental noise levels, the inherent absorption in the Earth and the random occurrence of small earthquakes have up to the present made any identification of spectral lines in the noise background impossible. The largest earthquakes (in particular. Chile in 1960 and Alaska in 1964) have led to signals sufficient to permit many tens of spectral peaks to be found (and used to refine Earth models). In the past year or two a new design of gravimeter at San Diego has shown free oscillations with a signal-to-noise ratio of up to $20 \mathrm{~dB}$ for many earthquakes down to magnitude 6.5 (in which the energy release would be several thousand times less than that for the Alaskan earthquake). And several other institutions report further free oscillations from new instrumentation responsive to ultra long periods and with low inherent noise levels-instruments which can reliably sense movements down to a few Ångstroms per hour are being looked for. But getting spectral lines out of the background on a day when no major event occurs, only smaller quakes, is a plum which many seismologists would like to pick, not just for fame but because seismic refinement of the structure of the Earth could then be a continuous process, not needing major earthquakes. Has Tuman pulled off this coup with his cryogenic gravimeter, certainly the most unusual instrument in geophysics today?

It is, of course, unfair to judge a conclusion that "four independent types of evidence indicate that spheroidal oscillations have been recordied continuously" solely on what is published. On the other hand, it can reasonably be assumed that the article puts as good a case as the author can make. On this basis, the assertions seem quite unjustifiable. Spectral analysis of 72 hours of random noise would produce a jagged output with a known mean and variance. There will be apparent spikes in the spectrum, but one can draw confidence limits around the mean and assert that these limits will not be exceeded more than so many per cent of the time. The statement that the signal ranges from 2 to $9 \mathrm{~dB}$ above the mean is unimpressive when random noise would produce similar fortuitous peaks, and a claim that several of the peaks are over the 95 per cent confidence limit invites the retort "yes, it looks like 5 are". Nothing in the data published suggests any deviation from a stochastic process. As has been noted, the density of spectral peaks of free oscillations increases rapidly towards the short period end, and this means that it will always be possible to pick an appropriate degree, order and overtone to fit any spectral peak. Thus simple association of peaks with modes does nothing to verify their reality with such poor signal-to-noise ratio. At longer periods the density of modes declines as does the density of sample points from a finite data window so the fit of peaks to modes should fall in quality, which indeed it does. There seems, however, to be just as many unexplained peaks at 800 seconds as there are at 400 seconds although the latter are called possible unknown overtones whereas the former are not mentioned. Does the spectrum continue in its jagged way up to the gravest mode and beyond, where if this were real free oscillation signal it should diminish to instrumental noise levels?
No data are shown. Equally curious is the identification of the gravest mode by its absence. This is claimed to be a result of the Earth's rotation in conjunction with the geographic location of the instrument. Because the small earthquakes assumed to be sustaining the free oscillations are presumably randomly located, the geographical location of the instrument could be assumed random and the earthquakes fixed in position, in which case there is no reason at all to expect rotational suppression of the gravest mode.

The least Tuman can be expected to do now is to show some of his seismograms. If the instrument really is sensing ground movement and its sensitivity is all that is claimed, the Earth tides should be a colossal feature, yet they are not mentioned.

It is decidedly distasteful to pick a public argument with a colleague, but Tuman's claims far exceed his published data, and the subject is, it has been noted, one in which there is a decidedly ripe plum for the winner.

\title{
Seeking Human Cancer Viruses
}

Although it has so far proved disappointing the search for human cancer viruses goes on apace. The direct approach is obviously to look in human cancer cells and in the body fluids of cancer patients for complete virus particles or for biochemical or immunochemical evidence of viral infection. So far this approach has revealed only two strong candidates: the Epstein-Barr herpesvirus, which is associated with Burkitt's lymphoma, and the B-type virus particles recently detected in some human milks (see Nature, 229, 593, 627 ; 1971), which in structure closely resemble the virus(es) which can cause breast cancer in mice. There is, however, a more subtle approach to the problem which has been used successfully for the isolation of animal cancer viruses, and in next Wednesday's Nature New Biology, McAllister and his six colleagues describe how they have exploited this in an attempt to isolate a human sarcoma virus.

The cat leukaemia and sarcoma viruses are no respectors of species barriers; they infect, and the feline sarcoma virus transforms, cells from a very wide range of species including man. Furthermore, the feline leukaemia virus is able to act as a so-called helper and rescuer of unrelated tumour viruses. For example, after infecting with feline leukaemia virus hamster cells transformed by a mouse sarcoma virus, progeny sarcoma virus is liberated, even though, before super-infection with the cat virus, the transformed cells did not produce any virus particles.
Apparently, the cat leukaemia virus provides the transforming mouse sarcoma virus, resident in hamster cells, with missing factors necessary for the production of progeny sarcoma virus.

This experimental, animal-cell system obviously indicates how cat sarcoma and leukaemia viruses might be used to reveal a human tumour virus. Following the reasoning of McAllister et al., suppose, for example, that human osteosarcoma cells are cancerous because they have been transformed by a human sarcoma virus, which is defective in the sense that it is unable to replicate and produce progeny virus particles. Such a cell would be equivalent to the hamster cell transformed by the mouse sarcoma virus. By superinfecting it with a mixture of cat leukaemia and sarcoma virus the defective human virus might be helped; in other words, the cat viruses might provide the missing functions necessary for the complete replication of the putative human sarcoma virus.

This is precisely the experiment that McAllister et al. have done; they superinfected with the two cat viruses a line of cells derived from a human osteosarcoma. After such transformation the growth properties of the cells are changed, they grow to greater densities, they require less serum and they also contain and yield virus particles. All the immunochemical and biological tests done so far on these progeny virus particles suggest they are identical to the feline leukaemia and sarcoma viruses used initially to superinfect the cells. 